\title{
The Text and the Context in Informal Analytics
}

\author{
Galina Sorina \\ Department of Philosophy of Language and Communication \\ Faculty of Philosophy \\ Lomonosov Moscow State University \\ Moscow, Russia \\ E-mail: gsorina@mail.ru
}

\begin{abstract}
This article presents the ideas behind informal text analytics (ITA) and the methodology for expert text analysis (META) as a tool of informal text analytics. The author addresses the interconnections between the text and the context in analytics. Immanuel Kant's ideas are considered as a methodological framework for ITA. At the same time, the article provides a comparative analysis of formal and informal analytics and emphasises the role of ITA in analytical practices and the possibility to hold a 'dialogue through centuries', particularly, with texts dating back to eras of the past. In this work, the author interprets the concept of text as any possible semiotic situation. The article stresses the decisive contribution made by Mikhail Bakhtin to formulating the theoretical problem of dialogue-based relations between the author and the reader. It is shown that there is always a conceptual framework behind a professional vision of the world.
\end{abstract}

Keywords-text; dialogue-based relations; formal analytics; informal analytics; text analytics; methodology for expert text analysis

\section{INTRODUCTION}

The philosophical and methodological approach to developing a framework for informal analytics is largely based on Kant's ideas about the methods for working with a text. Kant first formulated his methodological views, which will be interpreted below as those oriented towards informal text analytics, in the 'Announcement of the Programme of his Lectures for the Winter Semester 1765 - 1766' in 1765. In the 'Announcement', Kant addressed different methods for teaching philosophy. He stressed, The philosophical writer, for example, upon whom one bases one's instruction, is not to be regarded as the paradigm of judgement. He ought rather to be taken as the occasion for forming one's own judgement about him, and even, indeed, for passing judgement against him' [1. P. 293]. I believe that this holds true for not only a philosophical but also any other text. I am convinced that the ideas of informal text analytics (ITA) can be applied to analytics in different professional areas.

\section{THE HISTORY OF THE CONCEPT OF ANALYTICS AND ANALYTICAL PRACTICES}

Representatives of different fields of knowledge and different professions associate analytics primarily with a strictly formalised procedure. This idea of strictness is a result of a common perception of logic, namely, classical formal logic.

The idea of analytics as a strictly formalised procedure dates back to Aristotle's Analytics. In Prior Analytics, Aristotle defines the object of his inquiry as follows, 'it is about demonstration and ... its object is demonstrative science' [2. P. 1]. For Aristotle, demonstration or proof is a strict and formalised procedure. Aristotle considered developing a theory of the syllogism as the first step in producing a doctrine of demonstration. Within this theory, it is of no consequence who deliberates and in what context they do it [2]. Thus, the ideas of analytics merge with those of Aristotle's logic, namely, its part that was later called formal logic.

However, in the modern world, analytical practices do not meet the requirements of formal logic in the context of strict syllogistic reasoning. Numerous forms of analytics have been developed in different areas. For instance, there are such independent analytical research fields as mathematical analysis, logical analysis, logical analysis of language, political analysis, business analytics, legal analysis, international analysis, analytical support for education, and others. Analytics are becoming increasingly relevant in the rapidly changing world of today. Without analytics, it is impossible to put on hold the kaleidoscope of events. Moreover, the image of analytics, prevalent across different theoretical and practical fields of research, suggests that analytics is a strict, somewhat formalised, substantiated, and demonstrative means of reasoning.

The inherent contradiction of today's analytics is that explicit references to Aristotle's analytics are becoming obsolete, whereas the image of formalised subjectless procedures, which were an intrinsic part of Aristotle's syllogistics, persists. I believe it is necessary to stress that text analytics, despite various well-established protocols used in different areas, is an informal procedure. It depends on the contexts of texts and on the characteristics of agents who create texts and carry out analysis. The very idea of informal text analytics is emerging as a metaphor constructed by analogy with the ideas of informal logic and informal mathematics, in particular, Benoit Mandelbrot's informal geometry. 


\section{THE CONCEPT OF TEXT AND THE CLASSIFICATION PROBLEMS}

This article defines the text as any possible semiotic situation. At the same time, it provides a methodological framework for analysing written professional texts. Texts can be considered as a means of presenting a profession. Despite a detailed classification of texts used in different disciplines (historically, this has been a major concern of philology), the general rule is as follows - the more brilliant the text, the farther it goes beyond the boundaries established in its field of knowledge and the more complicated it is to analyse such a text in the context of classical text analysis schemes.

When the text is defined as any possible semiotic system, Euclidean geometry becomes an instance of the text. Moreover, in terms of ITA, the strict and formal nature of Euclidean geometry - when considered as a text - can generate a series of new texts constructed in accordance with Kant's approach, which was described above. This resulted in the emergence of Lobachevskian and Riemannian geometries. Lobachevskian geometry encouraged the creation of what I believe is one of the first forms of informal logic, namely, Vasiliev's imaginary logic [3]. Imaginary logic laid a foundation for the emergence of paraconsistent, many-valued, multidimensional, and intuitionistic logics. All of these new texts rest on an informal analysis of the text of Euclidean geometry. This leads to a 'spillover' of ideas from one professional area to another in the framework of ITA. The ideas of informal text analytics are becoming increasingly relevant in a rapidly changing world, where interdisciplinary and transdisciplinary studies are a common practice.

\section{EXPERT ANAlysis Methodology as a MeANS to IMPLEMENT THE ITA IDEAS}

An important tool to implement ITA ideas is the methodology for expert text analysis (META), which I developed and presented in a concise form in the work titled Expert text analysis: Methodology and practice [4].

Initially, the META was based on the work or dialogue with a text. 'For a modern person, who is immersed in the system of modern communication and the electronic culture, it is difficult to comprehend that ... the culture of printing lies at the heart of what we call classical culture. If one admits that the text is the substantial basis of that culture, from that moment on - although with certain reservations, one can speak of culture in general as the Text with a capital $\mathrm{T}^{\prime}[5]$.

One of the key characteristics of classical education is the commitment to free access to books and relevant commentaries and to the ability to think and analyse independently. It rests on systematic work with texts, primarily, written ones in both hard and soft copies.

Working with texts suggests a dialogue between the reader and the author. It does not matter when the text was written - each new reading brings a new life to it. Nor does it matter whether the text was released in soft or hard copy. Each time a classical text is read in the process of education, it raises question and creates a platform for dialogue including that 'through centuries'.

Texts addressing the same problem can represent different eras, positions, and perspectives. The primary goal of research - even student research - is to analyse different positions and hold an equal dialogue with them, taking into account when the text was written and when it is being read. It is important not only to learn and remember. It is necessary to be able to consider a variety of opinions to define one's own position.

Based on many years' experience of using the methodology for expert text analysis (META), it can be concluded that the META is not only an effective tool for modern educational communications but also a means to develop professional skills of knowledge workers in a knowledge society.

Any form of expert analysis requires a certain level of professionalism. At the same time, any expert analysis is based, to a degree, on examining different texts (for instance, legal, financial, economic, or political documents, different projects, programmes, agreements, and other field-specific documents).

The META suggests that, regardless of the context of a text and the area it to which it belongs, working with a text is based on a single technology stemming from the humanities. This technology lies at the heart of expert practices.

To perform expert work, one must be able to work with concepts, to enter a dialogue with the text (using an established Q\&A procedure), and to draw conclusions based on theoretical knowledge and using the system of associative links. All this can be presented in analytical reports (AR) communicating the results of studying texts in a variety of possible contexts. Moreover, ARs presenting the results of examining professional texts are prepared in the form of tables (see below). In effect, ARs based on the same text but prepared by several experts usually differ. Analytical reports prove the informal nature of expert analysis, despite the rigidity of AR submission forms. This occurs because questions about the text are formulated by the agents of analyses, depending on their knowledge, goals, etc. Only the first column shows similarities across reports prepared by different experts. Obviously, questions about texts, deliberations, comments, and the more so possible associations relating to the authors' professions, will be affected by their individual qualities and backgrounds. The structure of an analytical report is as follows "Table I".

TABLE I. The Structure of AN ANALYTICAL RePort

\begin{tabular}{|l|l|l|l|}
\hline $\begin{array}{c}\text { Key concepts } \\
\text { used in the text, } \\
\text { their major } \\
\text { characteristics }\end{array}$ & $\begin{array}{c}\text { Questions } \\
\text { about the } \\
\text { text }\end{array}$ & $\begin{array}{c}\text { Deliberations } \\
\text { and } \\
\text { comments }\end{array}$ & $\begin{array}{c}\text { Possible } \\
\text { associations } \\
\text { relating to the } \\
\text { author's } \\
\text { profession }\end{array}$ \\
\hline$\cdots$ & $\cdots$ & $\cdots$ & $\cdots$ \\
\hline$\cdots$ & $\cdots$ & $\cdots$ & $\cdots$ \\
\hline
\end{tabular}


Expert work sometimes offers a new vision of the text. A text analysis requires a search for missing information, the use of reference books, and immersion in the external information environment. The META is meant to be used in the practical analysis of texts existing in a variety of contexts.

Within the META, an analytical table provides a systematic framework for analysing the text in a variety of possible contexts.

\section{THE PROBLEM OF A 'DiAlogue Through Centuries'}

How does one enter into a dialogue with concrete researchers? How does one begin a conversation?

Mikhail Bakhtin made an invaluable contribution to formulating the theoretical problem of dialogue-based relations between the author and the reader/researcher. Bakhtin believed that research in the humanities is possible only as a thought about the thoughts and meanings of others and that it can be presented only in the form of a text. 'Regardless of the goals of a study, the starting point is always a text' [6. P. 124]. Texts are produced by agents who express their thoughts orally or in writing. For readers, foremost those striving to obtain a classical education, the conditions for entering into such a consistent dialogue can be laid down within a regular framework, for instance, that of the META. The methodology for text analysis is oriented primarily to a dialogue with a text and its author, regardless of the period when the text was written.

The idea of a 'dialogue through centuries' has inspired not only philosophers but also authors and poets. For instance, Osip Mandelshtam believed that 'nothing lyric is possible without a dialogue' [7. P. 149]. When discussing the problem of dialogue in lyrical poetry in his article 'On the interlocutor', Mandelshtam wrote, 'Exchanging whispers with your neighbour is boring, and drilling holes in one's own soul is infinitely dull. Trading signals with Mars there's a task worthy of a lyric poetry that respects the interlocutor... albeit individual poems, composed in the form of epistles or dedications, may address specific persons, poetry - as a whole - is ever moving toward that more or less distant, unknown addressee, whose existence the poet cannot doubt without also doubting himself. Only a reality can bring to life another reality' [7].

This long citation from Mandelshtam is meant to stress the significance of the problem of 'unknown addressee'. In effect, this very problem was analysed by Mandelshtam in the context of poetry in this 1913 article 'On the interlocutor'. Bakhtin pondered the same problem in the context of the humanities in 1959-1961 in his work 'The problem of the text'. A common research communicative space virtually enables the analyst of the next metalevel to construct a direct dialogue between, for instance, Bakhtin and Mandelshtam or Plato and the analyst's contemporary. A new research project focusing on a comparative text analysis is launched each time. This is the metalevel of analysis, which cannot be reduced to mere formal analytical apparatus. If one accepts Bakhtin's ideas, such a position of the researcher makes it possible to argue that each new situation in text analysis translates into an intellectual activity.

According to Bakhtin, not only the research situation but also each thought take on special characteristics and manifest themselves as an act. 'Every thought of mine, along with its content, is an act or deed that I perform-my own individually answerable act or deed [postupok]. It is one of all those acts which make up my whole onceoccurrent life as an uninterrupted performing of acts [postuplenie]. For my entire life as a whole can be considered as a single complex act or deed that I perform' [8. P. 3].

The methodology for expert text analysis is aimed to create a research situation each time a text is addressed and to ensure an understanding that this situation is essentially an act. At the same time, within expert work in a certain professional area, different forms of text analytics including formal and informal ones - can turn into a new project for the researcher. Understanding a text and text analysis become dependent on the professional status of a researcher.

Professional knowledge produces a distinctive vision of the world. For instance, the famous US economist, Paul T. Heyne, wrote 'The economic way of thinking employs such concepts as demand, opportunity cost, marginal effects, and comparative advantage to order familiar phenomena' [9]. Heyne admitted that such a vision of the world could distort the real picture but there was no other means to see the world. I believe it is necessary to stress that a professional vision of the world as a text requires a certain conceptual framework.

\section{CONCLUSIONS}

The methodology for expert text analysis rests on several foundations. These are as follows.

- Text analytics as a principal historical tool for the development of science and education, including the processes of their mutual influence.

- Expert analysis and decision-making in different theoretical and practical fields.

- The Socratic dialogue between everyone engaged in practical activities, including the instructor as a key participant in the dialogue and the main expert in text analysis.

- Commitment of the agents of educational processes to using IT in working with both classical and media texts, using the classical approach to text analysis and employing the classical apparatus of critical thinking.

- Commitment to teamwork principles in organising educational and research process.

- Teamwork projects.

- Understanding the effect of the researcher's individual qualities, professionalism, and background on expert work. 
Working with a text activates a structural vision of different field-specific and interdisciplinary problems. Moreover, it contributes to the development of a transdisciplinary approach to problems under considerations, which makes it possible to take into account relevant contexts and the characteristics of agents engaged in research. The universal nature of the methodology stems from a common framework for cognitive procedures and common teamwork principles.

In particular, this methodology - when applied to the system of education - meets the requirements of:

- project-based learning, often called experiential learning;

- the modern society, which places emphasis on independent decision-making and expert analysis, since almost any - even a technology - project has a context that relates to social sciences and the humanities and it always has consequences for these fields.

Expert work helps to develop skills necessary for a reflective analysis at both individual and collective levels, as well as responsibility for the outcomes of one's intellectual activities.

Employing the META in learning suggests that it helps students to take a more active part in projects and research and to obtain new knowledge within interdisciplinary and transdisciplinary studies. This can be done using the computer technology. In this context, it is possible to say that the META can serve as an intellectual tool used in training a new generation of labour force. It can also contribute to the efficiency of research, including that carried out by students. The methodology can be considered as a tool for implementing the ideas of informal text analytics. The study presented in this article has been supported by the Russian Foundation for Basic Research through the project 'Informal text analytics: A philosophical and methodological approach', No. 17-03-00772.

\section{REFERENCES}

[1] Kant, I. 'Immanuel Kant's Announcement of the Programme of his Lectures for the Winter Semester 1765 - 1766'. In D. Walford (Ed.), Theoretical Philosophy, 1755-1770 (The Cambridge Edition of the Works of Immanuel Kant, pp. 287-300). Cambridge: Cambridge University Press, 1992.

[2] Aristotle. Prior analytics. Hackett Publishing, 1989

[3] Vasiliev N.A. 'Imaginary logic.' Selected works. Moscow: Nauka, 1989.

[4] Sorina G.V. Expert text analysis: Methodology and practice. Moscow: IET, 2017.

[5] Mironov V.V. 'Literacy and book printing as a foundation of classical culture'. Space and Time electronic almanac, 2015, 10, 1.

[6] Bakhtin M.M. 'The problem of the text: A philosophical analysis'. Problems of literature, 10, 1976, pp. 122-151.

[7] Mandelshtam O.E. 'On the interlocutor'. (Accessed 21.05.2017). http://thebatterseareview.com/critical-prose/154-on-the-interlocutor

[8] Bakhtin M. Toward a philosophy of the act. University of Texas Press, 1993.
[9] Heyne P. The economic way of thinking, Science Research Associates, 1973. 